Bangladesh Journal of Neuroscience 2013; Vol. 29 (2) : 134-138

\title{
A Young Male with Typical Presentation of Amyotrophic Lateral Sclerosis (ALS)
}

\author{
SUKUMAR MAJUMDAR ${ }^{1}$, ANIS AHMED ${ }^{2}$, MD MASUD RANA $^{2}$, MAHFUZAR RAHMAN $^{3}$ \\ TAUHIDUL ISLAM ${ }^{4}$, MD SHAFIQUS SALEHEEN $^{5}$
}

\begin{abstract}
:
Amyotrophic lateral sclerosis (ALS) is a progressive neuromuscular condition characterized by proximal and distal muscle wasting, weakness, fasciculation. The etiology of the disease is unknown. The annual incidence rate is one to two cases per 100,000 persons. The disease is most commonly presents in middle age, mostly after the age of 50 and very uncommon before the age of 30 years and affects men more than women. Usually it present with limb muscle weakness, cramps, occasionally fasciculations and sometimes with dysarthria, dysphagia. Symptoms often begin focally in one part and spread gradually but relentlessly to become widespread. Over a period of months or years, patients with ALS develop severe, progressive muscular weakness and other symptoms caused by loss of function in both upper and lower motor neurons. Sphincter control, sensory function, intellectual abilities and skin integrity are preserved. Patients become completely disabled, often requiring ventilatory support and gastrostomy. Death usually occurs within five years of diagnosis and is attributed to respiratory failure or cachexia. Current management involves aggressive, individualized alleviation of symptoms and complications. We are reporting an 18 year old right handed male presented with typical features of ALS. The purpose for reporting was for its rarity before 40 years and that had a typical clinical features of young-adult ALS, and to compare them with features of the common adult-onset type.
\end{abstract}

Introduction:

Amyotrophic lateral sclerosis (ALS) was first described in 1869 by the French neurologist JeanMartin Charcot. It became familial after the popular baseball player Lou Gehrig was diagnosed with the disease in 1939 and best known as Lou Gehrig's disease ${ }^{1}$.It is a fatal neuro degenerative disease characterized by progressive muscular weakness reflecting degeneration of motor neurons in the motor cortex, brainstem and spinal cord ${ }^{2,3}$. It leads to muscle weakness and wasting due to unsent messages to muscles. The cause of ALS is unknown, although $5-10 \%$ of cases are familial.

The age of onset is usually after the age of 50 and very uncommon before the age of 30 . Juvenile 'ALS' refers to those with symptoms onset consistently before age 25 years, typically in association with a positive family history and slow progression ${ }^{5}$. Three genotypes of juvenile ALS have now been described. ALS2 (infantile ascending hereditary spastic paraparesis) ${ }^{6}$ shows autosomal recessive inheritance with very slow progression associated with a loss of function of the gene alsin and to date nine different mutations have been identified ${ }^{7}$. There is early onset of limb and facial muscle weakness accompanied by bulbar or pseudobulbar symptoms and upper motor neuron features predominate. ALS is associated with mutations in SETX ${ }^{8}$ and also known as distal hereditary motor neuronopathy with pyramidal features, shows autosomal dominant inheritance.

ALS is linked to chromosome 15 which is characterized by onset in the first or second decade of life with slowly progressive weakness and atrophy

1. Registrar, Department of Neuro-medicine, Rangpur Medical College and Hospital, Rangpur.

2. Medical Officer, Department of Neurology, Bangabandhu Sheikh Mujib Medical University (BSMMU), Dhaka.

3. Asistant Professor, Department of Medicine, Rangpur Medical College and Hospital, Rangpur.

4. Registrar, Department of Medicine, Rangpur Medical College and Hospital, Rangpur.

5. Indoor Medical Officer, Rangpur Medical College and Hospital, Rangpur. 
of the hands and feet and only later bulbar muscles ${ }^{5-}$ 9 . Upper motor neuron features are a late development ${ }^{10}$. 'Young-onset' Amyotrophic Lateral Sclerosis is considered to be similar to 'classic' ALS with mixed upper and lower motor neuron features commencing before an arbitrary cut-off age of 45 years and apparently sporadic. There is potential overlap of young onset with juvenile ALS in terms of age of onset, but cases of classical ALS with onset 20 years are exceptional ${ }^{11}$ and may indicate a different condition.

Males are more commonly affected than females ${ }^{12}$. The incidence is about $1-2$ per $100,000^{13}$. ALS affects motor neurons at 2 or more levels supplying multiple regions of the body. It affects lower motor neurons that reside in the anterior horn of the spinal cord and in the brain stem; corticospinal upper motor neurons that reside in the pre-central gyrus and pre-frontal motor neurons that are involved in planning or orchestrating the work of the upper and lower motor neuron. Loss of lower motor neurons leads to progressive muscle weakness and wasting (atrophy). Loss of corticospinal upper motor neurons may produce spasticity and abnormally active reflexes.

The term classic amyotrophic lateral sclerosis is reserved for the form of disease that involves both upper and lower motor neurons. The classic form of sporadic ALS usually starts as dysfunction or weakness in one part of the body and spreads gradually within that part and then to the rest of the body. If only lower motor neurons are involved, the disease is called progressive muscular atrophy (PMA). When only upper motor neurons are involved, the disease is called Primary lateral sclerosis (PLS). Rarely, the disease is restricted to bulbar muscles, in which case it is called progressive bulbar palsy (PBP). In most patients who present with initial involvement of bulbar muscles, the disease evolves to classic ALS.

Worldwide, ALS occurs sporadically in $90-95 \%$ of cases and with Mendelian patterns of heredity (familial ALS) in 5-10\% of cases. Most familial ALS is inherited in an autosomal dominant pattern ${ }^{14}$.

The diagnosis of ALS is primarily clinical. Electrodiagnostic testing contributes to the diagnostic

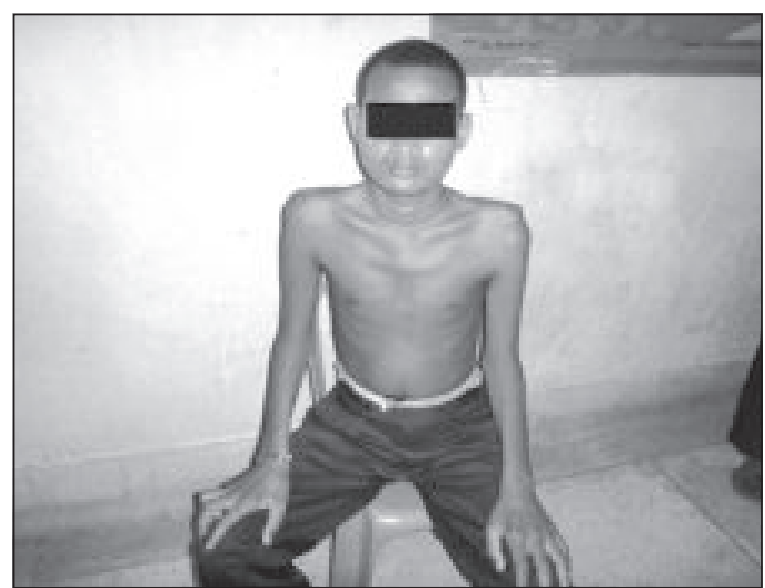

Fig.-1: The Patient with generalized muscle wasting

accuracy. It is relentlessly progressive; the mean time from diagnosis to death is 1 year, with most patients dying within 3-5 years of the onset of symptoms. Younger patients and those with early bulbar symptoms tend to show a more rapid course. Death is usually from respiratory failure, infection and the complications of immobility. Prognosis is better for progressive lateral sclerosis and progressive muscular atrophy. Here, we report a case of an amyotrophic lateral sclerosis of 18 years old man as because it is very uncommon before the age of 30 years.

\section{Case history:}

A 18 years old male, normotensive, non-diabetic college student hailing from Rangpur, Bangladesh admitted in Rangpur Medical College hospital, Rangpur, Bangladesh presented with progressive weakness and wasting of all four limbs for six months and slurring of speech for three months. Weakness first appeared in lower limbs and then gradually involved the upper limbs over three months associated with progressive muscle wasting. Weakness and wasting was bilaterally symmetrical involved both proximal and distal part of all four limbs equally. Patient also gave history of muscle twitching in different part of his body. He complained slurring of speech for last three months which also gradually increased associated with dysphonia and nasal regurgitation of liquid but no complain of numbness, tingling sensation or paresthesia in any part of his body. There was no history of fever, headache, 
unconsciousness or trauma to the head and neck. His bowel and bladder habit is normal. No member of his family suffered from such type of illness and no history of parental consanguinity.

General physical examination was unremarkable except the patient was emaciated. On neurological examination higher mental function was normal except speech which was slurred. Regarding cranial nerves examination- jaw jerk was present, palatal movement was diminished bilaterally and gag reflex was absent. Wasting with fasciculation of tongue observed but other cranial nerves were intact. There was symmetrical wasting of muscles of all four limbs but distal wasting was more than proximal. Wide spread fasciculations were observed in both calves, quadriceps, hamstring, gluteal muscles of lower limbs and biceps, triceps, deltoids, pectoralis, supraspinatus muscles of upper limbs. Tone slightly increased in both lower limbs but reduced in both upper limbs. Muscle power was $3 / 5$ in both upper limbs and $2 / 5$ in both lower limbs. Biceps, triceps and supinator jerks of both upper limbs were exaggerated but knee and ankle jerks of both lower limbs were diminished. Plantar response was extensor bilaterally. Coordination and gait was impaired. Sensory system found intact. Signs of meningeal irritation were absent. The laboratory investigations reports are summarized below-

TC of WBC $8,500 / \mathrm{mm}^{3}$, DC of WBC N-76\%, L15\%, M-3\%, E-4\%; Hb\%- $12.3 \mathrm{gm} / \mathrm{dL}$, Platelet count- 3.5 lacs $/ \mathrm{mm}^{3}$, ESR- $34 \mathrm{~mm}$ in the $1^{\text {st }}$ hour; RBS $6.6 \mathrm{mmol} / \mathrm{L}$; S creatinine $0.7 \mathrm{mg} / \mathrm{dl}$; S. bilirubin $0.7 \mathrm{mg} / \mathrm{dl}$, AST $47 \mathrm{U} / \mathrm{L}$, ALT $26 \mathrm{U} / \mathrm{L}$, Alkaline phosphatase $99 \mathrm{U} / \mathrm{L}$; S. electrolyte $\mathrm{Na}^{+} 142 \mathrm{mmol} /$ $\mathrm{L}, \mathrm{K}^{+} 3.9 \mathrm{mmol} / \mathrm{L}$; Fasting lipid profile- Cholesterol $176 \mathrm{mg} / \mathrm{dl}$, TG $281 \mathrm{mg} / \mathrm{dl}$, HDL $44 \mathrm{mg} / \mathrm{dl}$ and LDL $93 \mathrm{mg} / \mathrm{dl} ; \mathrm{FT}_{4} 1.18 \mathrm{ng} / \mathrm{dl}, \mathrm{TSH} 2.17 \mathrm{IU} / \mathrm{ml}$; VDRL non-reative; HBsAg (-)ve; ANA(-)ve; serum Vit $B_{12}$ level $631 \mathrm{pgm} / \mathrm{ml}$, serum Folic acid level $3.99 \mathrm{pgm} /$ $\mathrm{ml}$; MRI of cervical spine and brain reveals normal study; EMG showed diffuse neurogenic degeneration in the form of active denervation of bulbar and thoracic myotomes and active denervation with chronic reinnervation of cervical and lumbosacral myotomes.

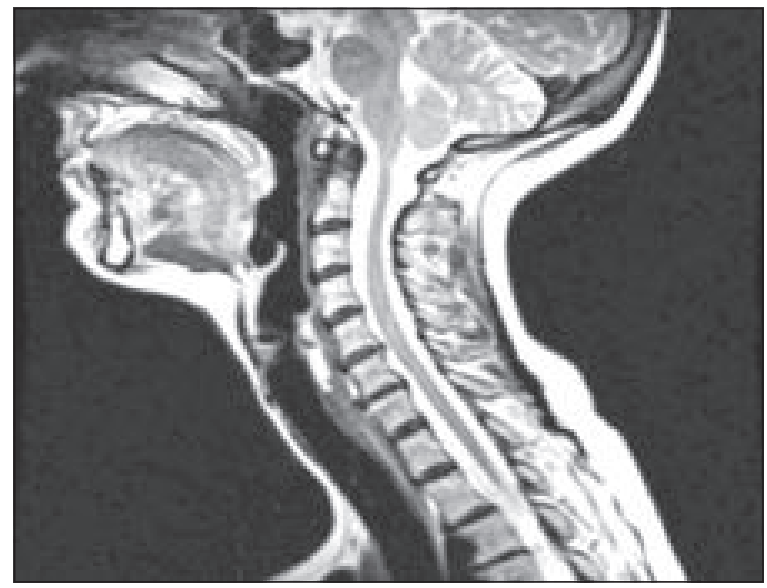

Fig.-2: MRI of Cervical spine of the patient showing normal anatomy.

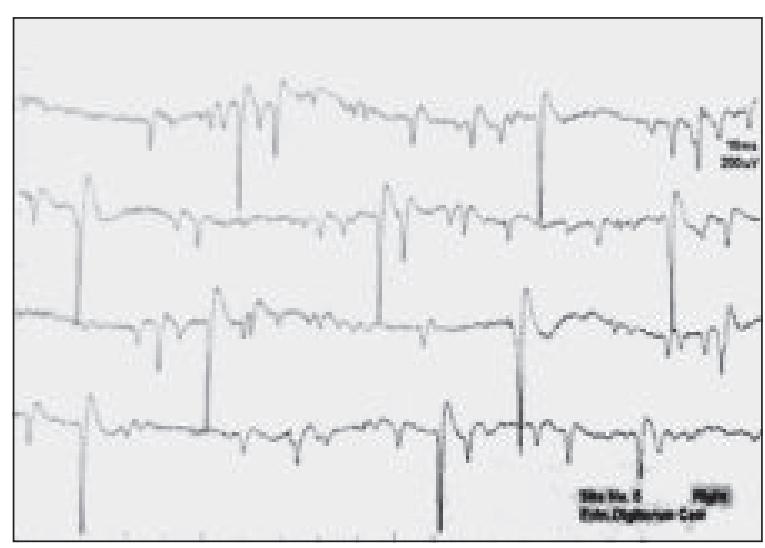

Fig.-3: EMG tracing of the patient

\section{Discussion:}

Amyotrophic lateral sclerosis (ALS) is a progressive and devastating neuro-degenerative disorder that affects motor neurons. It is the most common type of motor neuron disorder and does not lend itself to a quick definitive diagnosis early in its presentation. Often, neurologists need many months to exclude all other possible diagnoses in a patient presenting with upper and lower motor neuron signs. The characteristic form of this disease features the simultaneous presence of both upper motor neuron (UMN) and lower motor neuron (LMN) lesion signs, with progression from one region of the neuraxis to the next. Many cases of ALS will begin with the LMN form and then with time progress to show UMN involvement. Most ALS is sporadic and men tend to develop ALS more often than women with a male/ 
female ratio of about $2: 1^{15}$. Death, usually from respiratory compromise, occurs approximately three years after onset of symptoms ${ }^{16}$.Because of the near-uniform 'kiss of death' implications that a diagnosis of ALS carries, all efforts must be sought to exclude alternative diagnoses. In addition to electro-diagnostic studies, investigations usually include neuro-imaging studies to exclude anatomic structural processes such as cervical myelopathies and typical laboratory investigations to search for any potential treatable metabolic abnormality. Nerve conduction studies and needle electromyography are useful for confirming the diagnosis of ALS and for excluding peripheral conditions that resemble ALS. Laboratory test results generally are normal and are performed primarily to rule out other disease processes. The most common laboratory tests done are for serum vitamin B12 levels (to rule out subacute combined degeneration), parathyroid hormone levels (to rule out hyperparathyroidism) and serum protein electrophoresis with immune-fixation (to rule out multiple myeloma or MGUS). Imaging studies need to be tailored to the clinical presentation. Neuro-imaging may include computed tomography (CT) scan or magnetic resonance imaging (MRI) of the brain and spinal cord. These studies may be helpful to rule out structural lesions or neurological conditions that may mimic ALS. Examination of cerebrospinal fluid usually is not necessary. Genetic testing may be performed to identify gene defects in some familial types of ALS as well as other inherited motor neuron diseases ${ }^{17}$.

Our patient is a sporadic case. The patient typically presented with weakness and wasting of all four limbs and slurring of speech but no bowel and bladder involvement. On physical examination, he was found emaciated. On neurological examination, speech was found slurred, bulk of the muscles of all four limbs were reduced, tone was increased in both upper limbs but reduced in both lower limbs, muscle power were $3 / 5$ in upper limbs and 2/5 in lower limbs, deep tendon reflexes were absent in both upper limbs and exaggerated in both lower limbs, plantar responses were extensor bilaterally. Sensory system was found intact \& signs of meningeal irritations were absent .The diagnosis of ALS is clinical, based on the characteristic signs of progressive weakness, atrophy, fasciculations and hyperreflexia affecting several regions of the body. So, our patient's all clinical features are consistent with ALS. To establish the diagnosis of ALS beside the consistent clinical features one must have to exclude the probable differential diagnosis.EMG of the patient showed diffuse neurogenic degeneration in the form of active denervation of bulbar, cervical, thoracic and lumbosacral myotomes. The differential diagnosis may include musculoskeletal, neurologic or systemic conditions. Here we have excluded some possible differential diagnosis by laboratory investigations and imaging like Spinal cord lesions(tumors, syringomyelia, vascular malformations), Spinal bone lesions (spondylosis, cervical rib, metastatic tumors), infections (syphilis), Vitamin $B_{12}$ deficiency, endocrine disorder (hyperthyroidism, hyperparathyroidism, DM) and connective tissue disease (SLE).Genetic testing may be performed to identify gene defects in ALS but it is not available here.

The management of ALS is a complex and demanding team effort requiring individualized therapy and continual adaptation of medications and therapies. The only agent currently labeled for the treatment of ALS is riluzole which believed to decrease glutamate release. One large study reported that 56.8 percent of patients treated with $100 \mathrm{mg}$ of riluzole daily were alive without tracheostomy after 18 months, compared with 50.4 percent of patients who received a placebo, a clinically small but statistically significant difference ${ }^{18}$. But no study has reported that the drug halts the disease process ${ }^{19}$. Adverse effects include asthenia, nausea, dizziness, elevation of liver enzymes and granulocytopenia ${ }^{20}$.

We have prescribed riluzole $50 \mathrm{mg} 12$ hourly to our patient. Patient and his family was properly educated and counseled about the disease. The patient referred to the department of Physical Medicine and Rehabilitation of Rangpur Medical College and Hospital, Rangpur, Bangladesh, for proper physiotherapeutic management. We discharged him on request and advised to continue physiotherapy and follow up. 


\section{Conclusion:}

ALS is a fatal disease, with median survival of 3-5 years. Our findings showed that ALS can occur in younger age group and predominantly upper limb involvement. As this group represent a distinctive clinical variant, it needs to evaluate phenotypically, which was not done in this case due lack of facility of this test. Although ALS is incurable, the management involves aggressive, individualized alleviation of symptoms and complications that can prolong meaningful quality of life; therefore, diagnosis is important for patient and families.

\section{References:}

1. King SJ, Duke MM, O'Connor BA. Living with amyotrophic lateral sclerosis/motor neuron disease (ALS/MND): decision-making about 'ongoing change and adaptation'. J Clin Nurs. Mar 2009;18(5):745-54.

2. Wijesekera P, Nigel PN. Amyotrophic lateral sclerosis. Orphanet J Rare Dis. 2009; 4: 3.

3. Rowland LP, Shneider NA. Amyotrophic lateral sclerosis. N Engl J Med, 2001; 344: 16881700.

4. Allen CMC, Lueck CJ, Dennis M. Neurodegerative diseases. In: Colledge NR, Walker BR, Ralston SH, eds. Davidson's principles \& practice of medicine. Edinburgh: Elsevier, 2010; 1196-1205.

5. Roban A, Conwit T. Preventing familial ALS: A clinical trial may be feasible but is an efficacy trial warranted. Journal of the Neurological Sciences 2007; 251(2): 61-62.

6. Hadano S, Ricci C, Lotti EM, Benigni M, Gagliardi S, Zucco R. Severe familial ALS with a novel exon 4 mutation (L106F) in the SOD1 gene. Journal of the Neurological Sciences 2001; 293(1): 112-115.

7. LI J, Johnson M, Mandrioli N, Benatar M.Abramzon. Exome Sequencing Reveals VCP Mutations as a Cause of Familial ALS. Neuron 2011; 68(5): 857-64.

8. Blair F,Chen A. Frequency of the C9orf72 hexanucleotide repeat expansion in patients with amyotrophic lateral sclerosis and frontotemporal dementia: a cross-sectional study. Lancet Neurology 2000; 11(4): 323-30.

9. Gouveia D, Carvalho N, Bruijn L, Houseweart $\mathrm{M}$, Kato S, Anderson K. Aggregation and motor neuron toxicity of an ALS-linked SOD1 mutant independent from wild-type SOD1. Neuroscience 2007; 21(5): 18-24.

10. Eisen A. Amyotrophic lateral sclerosis: a 40year personal perspective. J ClinNeurosci 2009; 16: 505-512.

11. Armon C. Epidemiology of ALS/MND. In: Shaw P and Strong M, eds. Motor Neuron Disorders. London: Elsevier Sciences 2003; 167-206.

12. Walton JN, ed. Brain's Diseases of the nervous system. 10th ed. New York: Oxford University Press, 1993; 443-9.

13. Alonso A, Logroscino G, Jick SS, Hernán MA. Incidence and lifetime risk of motor neuron disease in the United Kingdom: a populationbased study. Eur J Neurol, 2009;16(6): 745-51.

14. Lacomblez L, Bensimon G, Leigh PN, Guillet $P$, Meininger V. Dose-ranging study of riluzole in amyotrophic lateral sclerosis. Lancet, 1996; 374:1425-31.

15. Bensimon G, Lacomblez, Meininger V. A controlled trial of riluzple in amyotrophic lateral sclerosis. N Engl J Med, 1994; 330(9): 585-91

16. Yanagisawa N, Tashiro K, Tohgi H. Efficacy and safety of riluzole in patients with amytrophic lateral sclerosis: double-blind placebo-controlled study in Japan. Igakuno Ayumi, 1997; 182: 851-866.

17. Rowland LP. Riluzole for the treatment of amyotrophic lateral sclerosis- too soon to tell? N Engl J Med, 1997; 330: 636-7.

18. Mitsumoto $\mathrm{H}$, Chad DA, Pioro EP. Amyotrophic lateral sclerosis. Philadelphia: Davis, 1998;16-24.

19. Oliver D. Motor neurone disease. 2nd ed. London: Royal College of General Practitioners, 1994;1-16.

20. Belsh JM, Schiffman PL. Amyotrophic lateral sclerosis: diagnosis and management for the clinician. Armonk, N.Y. Futura,1996; 286-90. 